\title{
Mini-Review \\ Performance Optimization in Sport: A Psychophysiological Approach
}

\author{
Selenia di Fronso ${ }^{1 *}$, Claudio Robazza ${ }^{1}$, Laura Bortoli ${ }^{1}$, Maurizio Bertollo ${ }^{1}$ \\ ${ }^{1} B I N D$-Behavioral Imaging and Neural Dynamics Center, Department of Medicine and \\ Aging Sciences, "G.d'Annunzio” University of Chieti-Pescara, Chieti, Italy.
}

\begin{abstract}
In the last 20 years, there was a growing interest in the study of the theoretical and applied issues surrounding psychophysiological processes underlying performance. The psychophysiological monitoring, which enables the study of these processes, consists of the assessment of the activation and functioning level of the organism using a multidimensional approach. In sport, it can be used to attain a better understanding of the processes underlying athletic performance and to improve it. The most frequently used ecological techniques include electromyography (EMG), electrocardiography (ECG), electroencephalography (EEG), and the assessment of electrodermal activity and breathing rhythm. The purpose of this paper is to offer an overview of the use of these techniques in applied interventions in sport and physical exercise and to give athletes, coaches and sport psychology experts new insights for performance improvement.
\end{abstract}

Keywords: performance improvement, psychophysiological approach, MAP model.

\section{Introduction}

Striving for success in practice and competition can be really struggling for athletes. It is not easy for them to attain excellent results during important events, especially when extended over long periods. In these situations, sport psychologists can help athletes prevent underperformance and/or find other ways to reach and maintain peak performance (i.e., optimal or outstanding achievements). Peak performance is an umbrella term used to classify positive states surrounding performance ${ }^{1}$ which includes concepts such as peak experience (typified by feelings of happiness and fulfillment) and/or flow (total absorption in a rewarding experience). Improving and reaching optimal performance during training and competition is one of the main goal for coaches and athletes. Unfortunately, optimal psychophysiological and psychosocial states do not occur frequently; competitive pressure can disrupt task execution and dysfunctional unpleasant states can appear. It is therefore necessary to implement self-regulation strategies to help performers cope with pressure and with dysfunctional feelings and/or emotions during these situations ${ }^{2}$.

We strongly advocate the development and adoption of idiosyncratic approaches for performance enhancement. Individualized strategies are also a main focus of the individual zones of optimal functioning (IZOF) model in which pleasant and unpleasant emotional states can exert beneficial, detrimental, or both effects on performance, depending on their individual meaning and intensity ${ }^{3}$.

Drawing on the IZOF model, Robazza and colleagues ${ }^{4}$ recently proposed the multi-action plan (MAP) model, an idiosyncratic approach aimed to enhance and optimize performance based on both emotion and action self-regulatory strategies. This model, in particular, places emphasis on the interaction between performance level and attentional control level during task execution. This $2 \times 2$ interaction (i.e., high/ low performance $\times$ high/low control level), defines four different types of performance-related states, in which athletes can experience functional or dysfunctional performance associated with pleasant or unpleasant states. Specifically, Type 1 performance is characterized by automatic movement execution, action "supervision", and optimal performance. Type 2 performance is typified by a higher level of attentional focus and functional performance. Compensatory resources need to be recruited for this performance state that can be obtained by consciously focusing attention on the core components of the action, such as pedaling rate in cycling 5 , braking point in car race $^{6}$, or aiming in shooting ${ }^{7,8}$. Type 3 suboptimal performance is characterized by an excessive attention focus that leads to over-controlled task execution, movement disruption, and dysfunctional performance. Type 4 performance is characterized by lack or task-irrelevant attentional focus and dysfunctional performance.

Paying attention to the core components of the action should help the athlete to recover from suboptimal performance states. Moreover, an appropriate focus on the core components should facilitate the switch on a less consciously controlled and more automatic task execution, and increase flow experiences.

\section{Psychophysiological monitoring: A multidimensional and multimodal approach to investigate performance}

A comprehensive assessment of performers implies the knowledge not only of those strategies to reach and maintain good performance (i.e., emotion-regulation, attentional strategies, control on the core components, etc.), but also of the psychophysiological underlying mechanisms that support the efficiency of the system. The study of human behavior in a holistic perspective, based on the assumption that measuring the processes of body-mind interaction can shed light on the human mind $^{9}$, can improve the understanding of these mechanisms. Psychophysiology is not devoted to a better knowledge of isolated components of the body, but rather it is addressed to gain insight on the functioning of the entire organism (e.g., attention-effort-optimal performance relationship) for performance optimization ${ }^{10}$. Adopting 
a psychophysiological approach to monitor and improve human and athletes' performance is therefore one of the most useful ways to study the mechanisms of body-mind integration and interaction with the environment.

The psychophysiological monitoring consists in the assessment of the activation and functioning level of the body. In sport, it can be used to better understand the underlying processes of performance and to subsequently increase or optimize $i^{11}$. Techniques used for this type of monitoring include, for example, electromyography (EMG), electrocardiography (ECG), electroencephalography (EEG), as well as other simple techniques to measure electrodermal activity (EDA) or respiratory rhythm. Electromyography (EMG) is a technique to analyze muscular signals generated by physiological changes in the state of the muscle membranes. Surface electrodes are mainly used for the study of neuromuscular activation in postural tasks, functional movements, working conditions, or workout. The analysis of these types of data provides useful information to improve performance in motor and sport activities. From a psychophysiological point of view, muscle tension has often been associated with emotional experience, and there are some target muscles, such as trapezium, which are often monitored for information about the level of anxiety and general tension of the athlete. Electrocardiography (ECG) measures heart electrical signal and provides parameters such as Heart Rate (HR) or Heart Rate Variability (HRV; i.e., the time interval variation between two heartbeats). In order to simplify the use of these measures, a pletismograph is placed on the finger of a hand instead of traditionally connect electrodes to the chest. In this way, blood flow variations in the finger capillaries, that faithfully represent the heartbeat, are recorded. Nowadays, chest strains wireless data assessed with the use of a computer are also used to monitor heart rate.

Heart rate variability may vary in response to factors such as breathing rhythm, emotional states, anxiety and stress. Linear analysis of HRV provide indices considered expression of sympathetic and parasympathetic activity and of the balance between these two branches of autonomic nervous system ${ }^{9}$. Examples of these indices in the frequency domain are: Very Low Frequency (VLF), usually associated with temperature regulation; Low Frequency (LF), usually associated with blood pressure regulation; High frequency (HF), associated with respiratory influences. The ability of the body to modify its balance toward one or the other activity (sympathetic or parasympathetic) is very important for a good cardiac functioning and is a mechanism that tends to a dynamic balance. High HRV is associated with good heart health and best sports performance, while periods of mental stress can reduce $i^{12}$. Heart rate variability can be attributed to changes in parasympathetic control associated with breathing and can be used as a vagal-heart rate index. In detail, HF linked to respiratory frequency reflects variations in the vagal control and is thus used as a parasympathetic activity index. In the field of sport psychophysiology, there is a great interest in HRV as it relates to arousal: HF increases, indeed, under high stress and anxiety conditions, especially with focused attention and motor inhibition. Other kind of linear and non-linear HRV-related indices (e.g., time domain indices, Poincare plot-related indices) instead, are recently being widely used to adapt training sessions and prevent dysfunctional phenomena such as overreaching and or/overtraining syndrome ${ }^{13}$.

Electrodermal activity (EDA) represents the electrical conductance that can vary according to the skin sweating level. Since the sweat glands are controlled by the sympathetic nervous system, the assessment of skin conductance (skin conductance level and skin conductance response) through specific sensors is considered an indicator of psychophysiological activation. EDA has been used in different studies: from the baseline evaluation of activation levels to the study of emotions and characterization of best performances ${ }^{14}$. In most cases, changes in EDA occur as a set of responses mediated by the autonomic nervous system. Increments of skin conductance level (SCL) are due to increased activation also related to emotions and anxiety. Stimuli with strong positive or negative affective value or associated with decision making are able to evoke electrodermal responses. Munro and colleagues ${ }^{15}$ noticed that SCL increased during the execution of a task and suggested two possible explanations: 1) tasks require a lot of attention resources and this may be associated with a higher activation of the autonomic nervous system; 2) stress and emotions are responsible of the increased SCL rather than attention and effort.

Another useful physiological parameter is respiratory rhythm, usually evaluated through a strain gauge below the chest cage. During task execution, breathing alterations can often be observed. For example, a superficial respiration involves mainly shoulder muscles and not abdominals; in other cases, the athlete may hold the breath or, conversely, voluntarily increase the respiratory rate. Such variations, also influenced by anxiety and stress, have been associated with poor performances ${ }^{12}$. Typically respiratory rhythm analysis is associated with heart rate variability, and idiosyncratic ${ }^{6}$ respiratory techniques are used to develop specific heart rate self-regulation training ${ }^{16}$.

Surface electroencephalography (EEG) in the scalp is the mostly used neuro technique in sport setting to record the electrical potentials produced by pyramidal cells. These measurements are obtained through electrodes placed on the head according to standard systems of positioning. EEG studies showed that specific frequency bands of brain oscillation (alpha, beta, theta, etc.) are associated with precise mental states (calm, arousal, attention, etc). Indeed, a great deal of research demonstrated an association between particular electroencephalographic patterns and performance in specific cognitive tasks and activities. For this reason, electroencephalography is used as a high-resolution temporal technique to understand performance and its improvements in the context of sports sciences ${ }^{17}$.

\section{Psychophysiological monitoring investigations}

Psychophysiologists generally use a multidimensional and multimodal approach to investigate performance, integrating physiological measurements with behavioral and psychological data. Measures such as skin conductance or those derived from EEG, EMG and ECG are linked to cognitive processes such as attention and emotional states. The modulation of physiological parameters can be used to control and increase 
performance. For example, heart rate (increase and decrease) has been studied in relation to the cognitive processes involved in motor performance ${ }^{18}$. Lacey and Lacey ${ }^{19}$ have developed a theory called "stimulus intake/rejection": the stimulus intake, which corresponds to a state of focused attention on external stimuli, is associated with a heart rate decrease; on the contrary, a state of focused attention on internal stimuli is followed by a heart rate increase. According to Tremayne and Barry ${ }^{20}$, a decrease in heart rate seems to be a sign of changes in attention state. Radlo and colleagues ${ }^{21}$ also considered the direction of attention (internal vs external focus). An external focus of attention was associated with a pronounced heart rate decrease immediately before a task (shot), while internal focus was linked to increased heart rate. Better performance was obtained with the use of an external focus, associated with heart rate decrease. Other authors have also considered HRV indices in relation to cognitive processes. Cooke and colleagues ${ }^{22}$ observed how heart rate variability in LF increased under augmented pressure during golf putting. The authors attributed this result to the increase in respiratory volume under pressure. In this case, an increased heart rate variability in HF band is explained by its sensitivity to breathing rhythm changes. However, researches have not observed any change in HF, despite the increased pressure and perception of greater effort during the task. It seems that the impact of the physical and postural demands of the putting on cardiac measurements, even minimal, is higher compared to that of mental activity. Neumann and Thomas ${ }^{23}$, on the other hand, examined heart rate and respiratory activity in elite, experts, and beginner golfers; regarding heart rate, in addition to a lower heart rate in general, expert and elite golfers, compared to beginners, showed a pronounced heart rate decrease immediately before the putting due to a more functional external focus. Mullen and colleagues ${ }^{24}$ gave a different explanation of the meaning of HRV. They conducted a study on amateur golfers using a putting task performed with and without a concurrent distracting task. They found that HF was higher during the putting condition with distracting stimulus and attributed this result to breathing relaxation strategies adopted by participants that affected the high frequency component of HRV.

Regarding EDA, Tremayne and Barry ${ }^{20}$ reported SCL to be a reliable index to monitor arousal/activation in precision sports. Electrodermal activity data showed a decrease in the values of expert shooters before the shot; it could reflect a quiet state of the body in the last seconds before the shot, immediately followed by a return to the initial values. These data help to elucidate the complex perceptual/cognitive processes involved in the performance of elite pistol shooters. SCL was recently used to identify individual zones of optimal functioning and typify optimal-poor and controlled-automatic performances in the framework of the MAP model. Specifically, an increase of SCL during more controlled performances (Type2, Type3) compared to the automatic ones (Type1, Type4) was found. These results suggest the existence of different mechanism of energy regulation and mobilization as related to the different types of performance states; an alternative explanation relies on the concept of attention and stress in a controlled situation, which leads to an increased electrodermal arousal ${ }^{14}$.
Electromiography has been used to evaluate neural activation during fatiguing isometric motor tasks, or different muscular activity related to internal and external focus. In this regard, Zachry and colleagues ${ }^{25}$ showed that the accuracy of the throw in basketball players was greater with an external focus (toward the basket) compared to an internal one (toward the arm). In addition, electromyographic activity of some muscle groups was found to be slower with an external focus compared to an internal one. This suggests that an external focus could increase the movement economy and reduce the elements in the motor system that impede movement control and impair the outcome. Moreover, neuromuscular data obtained through EMG analysis in a study about motivational cues on performance, interestingly indicated that this kind of stimuli partially blocked the effects of fatigue on the central motor command; indeed, participants that were administered these stimuli were able to sustain or even increase the neural activation of the working muscles during the final moments of a fatiguing isometric motor task ${ }^{26}$.

\section{Electroencephalography and cortical activity in sport}

Regarding the monitoring of brain activity in sport setting, electroencephalography is the most used technique because it can be used also outside the laboratory environment. Indeed, some types of equipment make possible to collect data even in more ecological settings. This enables researchers to describe optimal pattern of cortical activity during performance in various sports, such as archery, shooting, or golf ${ }^{11}$.

Electroencephalography in shooting sports allows detecting optimal performance predictors through electroencephalographic differences in pre-shooting conditions between experts and novices, and between optimal and non-optimal performances in the same athlete. This technique allows the study of some brain wave characteristics and frequencies, linking them to specific situations (as mentioned before). In their review chapter on superior sport performance, Hatfield and Kerick ${ }^{11}$ observed that there was a cortical asymmetry in the temporal area in the alpha band of rifle shooters during a 7.5-sec interval before the shot. Alpha power showed a noticeable increase over the 7.5-sec interval in the left hemisphere, while only a slight increase was found in the right hemisphere. These authors interpreted the increase of alpha activity in the left hemisphere as a decreased activation of the same hemisphere. The same authors noticed that the left temporal hemisphere is devoted to language processing and suggested that increased alpha activity might indicate a reduction of the symbolic and verbal activity as well as of self-talk, and an allocation of more resources to the visual-motor stream in the right hemisphere. This interpretation seems to be congruent with the idea that self-talk can be a potential performance "disturbing" process.

An ecological approach to assess differences in skill level and the related mental states is useful to examine the differences between expert and non-expert athletes in a specific task. Del Percio and colleagues ${ }^{27}$ found that elite pistol shooters are characterized by an increase in the power of high alpha band (10-12 $\mathrm{Hz}$ ) and beta band (14-35 Hz), probably linked to attentional 
processes. In a subsequent study, results suggested that elite athletes are characterized by a stabilization in the functional coupling of preparatory waves (alpha-beta) between the frontal visual-spatial areas and parietal-occipital cortical areas ${ }^{28}$. Doppelmayr and colleagues ${ }^{29}$, on the other hand, have noticed a steady increase in theta power during the last 3 seconds before shot release only in expert pistol shooters but not in non-experts. The results indicated higher theta wave activity in expert athletes, closely localized in the anterior cingulate area and in the frontal cortex. Experts and non experts use different strategies during the aiming period. Indeed, novices keep a relatively constant amount of attention to the target, while experts are able to increase attention exactly to the time point of the trigger pull.

Electroencephalography was also used to study the influence of motivational audiovisual stimuli, such as music and video, in increasing situational motivation and enhance performance ${ }^{26}$. Specifically, it was demonstrated that sensory stimuli mediate brain responses to exercise that ameliorate the effects of fatigue and increase situational motivation. The motivational audiovisual stimuli seemed to modulate the amplitude of theta waves in the frontal cortex and beta waves in the central areas of the brain; the motivational content of the sensory stimulus likely influences the activity of the central motor command by decreasing low-frequency waves and consequently the fatigue, and increasing beta activity and the arousal level. The effects of the motivational stimuli on the central regions of the cortex are probably linked to the protective mechanisms of motivation on exercise engagement; in particular, motivational stimuli partially obstacle the negative effects of fatigue on psychophysiological responses and exercise performance.

More recently, Cheron and colleagues have described in depth the meaning of the oscillatory brain rhythms hypothesizing some specific EEG biomarkers ${ }^{30}$. Theta oscillation, for instance, plays an important function in motor control. It was also demonstrated that the theta power increase in the controlateral motor area during the onset of fast ballistic movements ${ }^{30}$. Moreover, theta oscillation was related with the onset of the movement and the theta power correlated with movement acceleration ${ }^{30}$. Other studies showed an association between different stages of exercising and modulations of the alpha rhythm. Despite contrasting results, it was shown that the shift from rest to exercise is linked to an increase in alpha band, while brain activity during and after exercise has been associated with both upward ${ }^{31}$ and downward variations ${ }^{31}$. This rhythm was also studied as a marker of resources allocation during motor programming ${ }^{32}$. Beta oscillation (15-30 $\mathrm{Hz}$ ), on the other hand, has a central position in the treatment of sensorimotor information and serves as a functional link between different brain regions such as the pre-motor, motor (M1), and somatosensory (S1) cortex, the supplementary motor area, and the cerebellum ${ }^{31}$. Beta oscillation could play the role of a motor "binding" linking the different commands related to a global gesture as suggested for sensory awareness ${ }^{30}$.

Current advances in EEG signal processing and modifications of the hardware led to EEG recordings while individuals behave naturally, like walking outdoors. It has also been demonstrated that wireless EEG can be used to collect data in a simulated working situation involving complex motor actions or in a cooperative motor task ${ }^{33}$. Moreover, highly portable new EEG systems (with dry and wet electrodes) could lead to major progresses in brain computer interfaces and neurofeedback protocols.

\section{Applied psychophysiology and the MAP model}

The MAP model predictions have been recently examined from a psychophysiological point of view to better understand the underlying psychophysiological processes and the usefulness of action and/or emotion centered strategies in reaching and maintaining optimal performance.

In order to test whether specific affective, psychophysiological, and postural trends could typify performance states as conceptualized in the MAP model, Bertollo and colleagues conducted a single-subject study on pistol shooting and dart throwing ${ }^{14}$.The shooter involved in the study showed lower levels of SCL in Type 1 performance compared to Type 2 and Type 3, and similar levels in Type1 and Type 4. A similar pattern also emerged in the dart throwing participant. SCL can be considered one of the best indicators of the arousal-activation binomial in these precision sports. A higher electrodermal activity in Type 2 and Type 3 performance states compared to Type 1 and Type 4 suggested the existence of different mechanisms of energy mobilization and regulation, and a related increased autonomic nervous system activation. No differences were observed on HR among the four types of performances likely because of fatigue that caused a generalized HR increase. The decrease in respiratory rate found in Type 3 performance suggested that the respiration process can be influenced by fatigue due to the attentional control exerted during execution. All these interpretations have potential practical implications. Coaches could monitor psychophysiological states of athlete, help them identify individualized performance optimization strategies, and use biofeedback techniques to improve self-regulation and monitor individual levels of arousal-activation.

The MAP model assumptions were also tested in motor sport and among professional racecar drivers. Filho and colleagues ${ }^{6}$ analyzed the kinematic and psychophysiological mechanism of braking modulation and acceleration dynamics. Findings supported the notion that different psychophysiological states underlie the different MAP model categories. From an applied point of view, given that most drivers showed unique heart rate and respiratory rate patterns linked to Type 1 performance, a heart rate variability training may increase the probability of peak performance experiences. Moreover, large individual differences among the drivers were observed, specifically in the intensity, variability, and magnitude of the drivers' subjective and psychophysiological recordings. These results are in accordance with the principle of individualization in athletic training and reinforce the importance of idiosyncratic studies and applications in sport psychology, especially among skilled athletes. Results also underlie the importance of developing idiosyncratic and multimodal plans, including bio-neurofeedback training, for performance optimization

Recently, EEG data collection and analysis (Event-Related Desynchronization/Synchronization-ERD/ERS analysis) were 
performed to better identify neural markers underlying each performance state. Findings in an Olympic shooting athlete ${ }^{7}$ further supported the MAP model tenets. Optimal-automatic (Type 1) and suboptimal-controlled (Type 3) states were indeed typified by distinct neural activity patterns. Specifically, EEG analysis showed a synchronization pattern in Type 1 performance in agreement with the neural efficiency hypothesis (i.e., optimal performance and low cognitive exertion), and desynchronization pattern in Type 3 likely due to verbal analytical processes disrupting movement automatisms. A task-relevant focus on the core components of the action can allow the athlete to regain an optimal performance state. Findings can inform neurofeedback training for performance enhancement. Results can also help the identification of the cortical areas where to apply transcranial stimulation methods ${ }^{34}$ to facilitate cortical plasticity.

The MAP model predictions were also examined in a singlesubject study using a cycling task ${ }^{35}$. Cortical functional connectivity was assessed through EEG coherence analysis, which has been extensively used to study the neural mechanisms underlying perceptive, cognitive (e.g., information exchange between brain areas), and motor processes ${ }^{28}$. Overall, results indicated specific functional connectivity patterns to be associated with different attention strategies; for instance, an extensive communication among areas was found in a Type 1 performance state prompted by an external associative strategy (i.e., attention focused on a metronome during the cycling task). Findings support the hypothesis that specific strategies may stimulate flow experiences characterized by specific electrophysiological patterns leading to better performance.

\section{Psychophysiological interventions: bio-neurofeedback and transcranial stimulation}

Psychophysiological monitoring provides a great amount of information on the athlete's performance-related states: heart rate, heart rate variability, brain waves, muscle activity, and autonomic nervous system data are crucial, as they provide a holistic view and a better understand of performance states. An important purpose of the psychophysiological monitoring is the use of bodily signals as a reliable feedback to help athletes improve their awareness of bodily functions and develop self-regulation skills. Through brain-body computer interface, biofeedback (BFB) and neurofeedback (NFB) systems display information that individuals can use to self-regulate brain-body processes in real time. Moreover, multiple types of signals can be integrated, thus advancing the understanding of complex performance factors and self-regulation systems.

BFB training with selected modalities (e.g., EMG and SCL) have been used to enhance motor performance and movement patterns in sport disciplines such as gymnastics and kayaking ${ }^{36}$. HR, EEG, and respiratory BFB training have been used for performance enhancement in aiming tasks (e.g., archery, shooting, golf); EMG and SCL BFB training is used also in combat sports (e.g., taekwondo, fencing, judo). BFB training includes learning and improving self-monitoring and self-regulation skills for performance enhancement also under competitive stress.
It can be integrated within elite level preparation as already demonstrated in various sports ${ }^{36}$. Positive effects of BFB training provide athletes with objective feedback regarding their self-regulatory skills.

NFB training is a specific type of BFB training that provides a feedback regarding one's cortical activity ${ }^{37}$. Positive effects of NFB have been found in aiming tasks, such as archery, shooting, and golf as well as in other sport disciplines ${ }^{37}$. NFB training can be used together with relaxation, and mental skill training, involving imagery and self-talk, to help athletes increase alpha or beta brain waves and therefore self-regulate their arousal level.

Beyond NFB training, there are other methods to improve skills and motor performance based on neuroplasticity ${ }^{38,39,40}$. Non-invasive brain stimulation (NIBS) techniques facilitate motor skill learning by increasing the corticospinal excitability. NIBS include, for instance, transcranial direct current stimulation (tDCS) and transcranial random noise stimulation (tRNS) ${ }^{34}$. Evidence from recent studies suggests links between tDCS induced corticospinal excitability, skill learning, and motor performance ${ }^{40}$. In a golf putting task, it was shown that tDCS applied over the left side of prefrontal cortex facilitates implicit motor learning. As regards tRNS, this is a form of stimulation applied at random frequencies between 0.1 and $640 \mathrm{~Hz}$, which can lead to an increase in performance of implicit motor or perceptual learning tasks ${ }^{40}$.

\section{Conclusion}

This review highlights the beneficial effects that can derive from the adoption of a psychophysiological approach for performance optimization. The multidimensional and multimodal monitoring facilitates an idiosyncratic assessment of athletes, and the development of individualized interventions using BFB and NFB training or stimulation methods. This method, combined with classical psychological interventions (relaxation, attentional strategies, etc.), is intended to enhance performance and execute consistently. Athletes can improve self-regulation skills, attain flow experiences and peak performance, and increase their general wellbeing. Furthermore, in the framework of MAP model a psychophysiological approach is suggested to improve the athletes' awareness of mental and emotional states underpinning the four performance types.

\section{References}

1. Kimiecik JC, Jackson, SA. Optimal experience in sport: A flow perspective. In: advances in sport psychology. Champaign, IL. Human Kinetics,2002. p.501-529.

2. Beckmann J, Gröpel P, Ehrlenspiel F. Preventing motor skill failure through hemisphere-specific priming: Cases from choking under pressure. J Experim Psychol: General. 2013;142(3):679-69. doi:10.1037/a00298521.

3. Robazza, C. Emotion in sport: An IZOF perspective. In: Literature reviews in sport psychology. Hanton S, Mellalieu SD (Eds). 2006; p.127-158. 
4. Robazza C, Bertollo M, Filho E, Hanin Y, Bortoli L. Perceived Control and Hedonic Tone Dynamics During Performance in Elite Shooters. Res Q Exerc Sport 2016;87(3):284-294. doi:10.1080/0 2701367.2016.1185081

5. Bertollo M, di Fronso S, Filho E, Lamberti V, Ripari P, Reis VM, et al. To focus or not to focus: is attention on the core components of action beneficial for cycling performance? The Sport Psychol. 2015;29(2):110-119. doi:10.1123/tps.2014-0046

6. Filho E, di Fronso S, Mazzoni C, Robazza C, Bortoli L, Bertollo M. My heart is racing! Psychophysiological dynamics of skilled racecar drivers. J sports sci.2015;33(9):945-959. doi:10.1080/0 2640414.2014.977940

7. di Fronso S, Robazza C, Filho E, Bortoli L, Comani S, Bertollo M. Neural markers of performance states in an Olympic Athlete: an EEG case study in air-pistol shooting. J sports sci \& med. 2016;15(2):214-22.

8. Bertollo M, di Fronso S, Conforto S, Schmid M, Bortoli L, Comani S, et al. Proficient brain for optimal performance: the MAP model perspective. PeerJ. 2016;4:e2082. doi:10.7717/peerj.2082

9. Cacioppo JT, Tassinary LG, Berntson, G, editors. Handbook of psychophysiology. Cambridge. University Press, 2007.

10. Tang YY, Bruya B. Mechanisms of mind-body interaction and optimal performance. Front Psychol. 2017;8:647. doi:10.3389/ fpsyg.2017.0064747.

11. Hatfield BD, KerickSE. The psychology of superior sport performance. In: Handbook of sport psychology. 3rd ed. Hoboken (NJ): Wiley, 2007. p.84-109.

12. Wilson WS, Somers K. Psychophysiological assessment and training with athletes. Knowing and managing your mind and body. In B. Strack, M. Linden, \& V. S. Wilson (Eds.), Biofeedback and neurofeedback applications in sport psychology Wheat Ridge, CO: American Association Psychophysiology \& Biofeedback. 2011. p. 45-88. doi:10.20381/ruor-533.

13. Proietti R, di Fronso S, Pereira LA, Bortoli L, Robazza C, Nakamura FY, et al. Heart Rate Variability Discriminates Competitive Levels in Professional Soccer Players. The J Strength \& Cond Res.2017;31(6):1719-1725. doi:10.1519/ JSC.0000000000001795

14. Bertollo M, Bortoli L, Gramaccioni G, Hanin Y, Comani S, Robazza C. Behavioural and psychophysiological correlates of athletic performance: A test of the multi-action plan model. Appl Psychophysiol Biof 2013;38(2):91-99. doi:10.1007/ s10484-013-9211-z

15. Munro LL, Dawson ME, Schell AM, Sakai LM. Electrodermal lability and rapid performance decrement in adegraded stimulus continuous performance task. J Psychophysiol. 1987;1:249-257.

16. Lagos L, Vaschillo E, Vaschillo B, Lehrer L, Bates M, Pandina R. Heart rate variability biofeedback as a strategy for dealing with competitive anxiety: A case study. Biof. 2008;36(3):109-115

17. Park JL, Fairweather MM, Donaldson DI. Making the case for mobile cognition: EEG and sports performance. Neurosci Biobehav Rev 2015;52:117-130. doi:10.1016/j.neubiorev.2015.02.014

18. Abernethy B, Maxwell JP, Masters RS, Van der Kamp J, Jackson RC. Attentional processes in skill learning and expert performance. In G. Tenenbaum \& R. C. Eklund (Eds.), Handbook of sport psychology New Jersey, NJ: Wiley. 2007. p. 245-263.
19. Lacey BC, Lacey J Cognitive modulation of time-dependent primary bradycardia. Psychophysiol. 1980;17:209-221. doi:10.1111/j.1469-8986.1980.tb00137.x

20. Tremayne P, Barry RJ. Elite pistol shooters. Physiological patterning of best versus worst shots. Int J Psychophysiol. 2001;41:19-29. doi:10.1016/S0167-8760(00)00175-6

21. Radlo SJ, Steinberg GM, Singer, RN, Barba DA, Melnikov A. The influence of an attentional focus strategy on alpha brain wave activity, HR, and dart-throwing performance. Int J Sport Psychol. 2002;33:205-217

22. Cooke A, Kavussanu M, McIntyre D, Ring C. Psychological, muscular and kinematic factors mediate performance under pressure. Psychophysiol. 2010;47:1109-1118. doi:10.1111/j.1469-8986.2010.01021.x

23. Neumann DL, Thomas PR. The relationship between skill level and patterns in cardiac and respiratory activity during golf putting. Int J Psychophys. 2009;72:276-282. doi:10.1016/j. ijpsycho.2009.01.001

24. Mullen R, Hardy L, Tattersall A. The effects of anxiety on motor performance: a test of the conscious processing hypothesis. J Sport \& Ex Psychol. 2005;27:212-225. doi:10.1123/jsep.27.2.212.

25. Zachry T, Wulf G, Mercer J, Bezodis N. Increased movement accuracy and reduced EMG activity as the result of adopting an external focus of attention. Brain Res Bull, 2005;67:304-309. doi:10.1016/j.brainresbull.2005.06.035.

26. Bigliassi, M, SilvaVB, Karageorghis CI, Bird JM, Santos PC, AltimariLR. Brain mechanisms that underlie the effects of motivational audiovisual stimuli on psychophysiological responses during exercise. Physiol \& behav. 2016;158:128-136. doi:10.1016/j. physbeh.2016.03.001

27. Del Percio C, Babiloni C, Bertollo M, Marzano N, Iacoboni $\mathrm{M}$, Infarinato $\mathrm{F}$, et al. Visuo-attentional and sensorimotor alpha rhythms are related to visuo-motor performance in athletes. Hum Brain Map.2009;30:3527-354. doi:10.1002/hbm.20776

28. Del Percio C, Iacoboni M, Lizio R, Marzano N, Infarinato F, Vecchio F, et al. Functional coupling of parietal alpha rythms is enhanced in athletes before visuo-motor performance: a coherence electroencephalographic study. Neurosci. 2011;175:198-211. doi:10.1016/j.neuroscience.2010.11.031

29. Doppelmayr M, Finkenzeller T, Sauseng P. Frontal midline theta in the pre-shot phase of rifle shooting: differences between experts and novices. Neuropsychol. 2008;46:1463-1467. doi:10.1016/j. neuropsychologia.2007.12.026

30. Cheron G, Petit G, Cheron J, Leroy A, Cebolla A, Cevallos C, et al.Brain oscillations in sport: toward EEG biomarkers of performance. Front psychol. 2016;7:246. doi:10.3389/fpsyg.2016.00246

31. Gutmann B, Mierau A, Hülsdünker T, Hildebrand C, Przyklenk A, Hollmann W, et al. Effects of physical exercise on individual resting state EEG alpha peak frequency. Neural plast. 2015. doi:10.1123/jsep.27.2.212

32. Cooke A, Gallicchio G, Kavussanu M, Willoughby A, McIntyre D, Ring C. Premovement high $\square$ alpha power is modulated by previous movement errors: Indirect evidence to endorse high $\square$ alpha power as a marker of resource allocation during motor programming. Psychophysiol. 2015;52(7):977-981. doi:10.1111/ psyp. 12414 
33. Filho E, Bertollo M, Tamburro G, Schinaia L, Chatel-Goldman J, di Fronso S. et al. Hyperbrain features of team mental models within a juggling paradigm: a proof of concept. PeerJ. 2016;4:e2457. doi: $10.7717 /$ peerj. 2457

34. Paulus W. Transcranial electrical stimulation (tES-tDCS; tRNS, tACS) methods. Neuropsychol rehab. 2011;21(5):602-617. Doi: 10.1080\09602011.2011.557292

35. Comani S, di Fronso S, Castronovo AM, Schmid M, Bortoli L, Conforto $\mathrm{S}$, et al. Attentional focus and functional connectivity in cycling: An EEG case study. In XIII Mediterranean Conference on Medical and Biological Engineering and Computing 2013. Springer, Cham. 2014.p. 137-140. doi:10.1007/978-3-319-00846-2_34

36. Blumenstein B, Tsung-Min Hung E. Biofeedback in sport. Routledge international handbook of sport psychology. 2016; p. $429-438$

37. Mirifar A, Beckmann J, Ehrlenspiel F. Neurofeedback as supplementary training for optimizing athletes' performance: a systematic review with implications for future research. Neurosc \& Biobehav Rev. 2017;75:419-432 doi:10.1016/j. neubiorev.2017.02.005

38. Tommasi V, Prete G, di Fronso S, Schinaia L, Lucafò C, Tommasi L, et al. The Effect of tRNS on Performance: a Pilot Study with a Skilled Air-Pistol Shooter. Biof. 2015;43(2):84-89. doi:10.5298/1081-5937-43.2.02

39. Vitor-Costa M, Okuno NM, Bortolotti H, Bertollo M, Boggio PS, Fregni F, et al.Improving cycling performance: transcranial direct current stimulation increases time to exhaustion in cycling. PloS one. 2015;10(12):e0144916. doi:10.1371/journal.pone.0144916

40. Colzato LS, Nitsche MA, KibeleA. Noninvasive Brain Stimulation and Neural Entrainment Enhance Athletic Performance-a Review. J Cog Enhanc. 2017;1:73-79. doi:10.1007/s41465-016-0003-2

\section{Corresponding author}

\section{Selenia di Fronso}

BIND-Behavioral Imaging and Neural Dynamics Center, Department of Medicine and Aging Sciences, "G.d'Annunzio" University of Chieti-Pescara, Chieti, Italy

Email: s.difronso@gmail.com

Manuscripted received on September 25, 2017

Manuscripted accepted on September 25, 2017

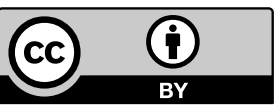

Motriz. The Journal of Physical Education. UNESP. Rio Claro, SP, Brazil - eISSN: 1980-6574 - under a license Creative Commons - Version 3.0 